\title{
Enhanced Properties of the Monomer and Co-Polymer with Broad Band Absorption
}

\author{
Nahla Omer ${ }^{1,3}$ and Hongyao $\mathrm{Xu}^{1,2 *}$ \\ ${ }^{1}$ College of Material Science and Engineering and State Key Laboratory for Modification of Chemical Fibers and Polymer Materials, Donghua \\ University, Shanghai, China \\ ${ }^{2}$ Research Center for Analysis and Measurement, Donghua University, Shanghai, China \\ ${ }^{3}$ Sudan University of Science and Technology (SUST) College of Engineering Department of Science, Sudan \\ *Corresponding Author: Hongyao Xu, College of Material Science and Engineering and State Key Laboratory for Modification of Chemical \\ Fibers and Polymer Materials, Donghua University, Shanghai, China.
}

Received: June 28, 2019; Published: July 10, 2019

DOI: $10.31080 /$ ASPS.2019.03.0335

\begin{abstract}
Nowadays organic dye monomer and co-polymer materials have been receiving more interested in many applications due to improved solution process ability, scalable synthesis, tunable chemical and physical properties via molecular design and low cost. However, the compatibility and aggregation of organic Squaraine dye and co-poly acrylamide limited broad application. In this study, we designed two systems enhanced by octavinyl-polyhedral oligomeric silsesquioxane (OV-POSS) which solved the compatibility and aggregation. These is the first time designed a novel two systems OV-POSS-Squaraine and OV-POSS-co-poly acryl amide with wide band absorption
\end{abstract}

Keywords: Broad Band Absorption Molecular Design Co-Polymer; Squaraine Dye; Compatibility; Aggregation

\section{Introduction}

Among all the organic dyes fixed, on squaraine (SQ) dyes are particularly promising class of dyes widely applied in solar cells, photovoltaic devices, and bio-imaging because possess many particular advantages such as easy molecular design, strong absorption coefficient, properties $(\varepsilon \geq 105 \mathrm{~L} \cdot \mathrm{mol}-1 \cdot \mathrm{cm}-1)$ from red to near-IR regions [1-4]. Generally, SQ dye absorbing the near infrared (NIR) region $550 \mathrm{~nm}$ to $900 \mathrm{~nm}$ and known as donor acceptor (D-A) [5]. However, these types of materials suffering from poor compatibility and aggregation, therefore, how to design well compatibility dye-molecular structure [6]. However also, there were many water-soluble monomers are used for synthesizing water-soluble fluorescent polymers, which includes N-Nisopropylacrylamide, N-dimethyl acrylamide [7] and polyacrylamide derivatives [8]. However, most of them suffer from the same problems of the dye. TheOV- POSS have been used to enhance the poor compatibility, aggregation and dielectric properties of organic dyes and co-polymer [9-15]. So in this study, to make full use of the design it is needful to use materials with broad band absorption, in one material it is rarely reported (Scheme.1- OV-POSS-SQ) and (Scheme. 2- OV-POSS-OV-POSS-copoly acryl amide).

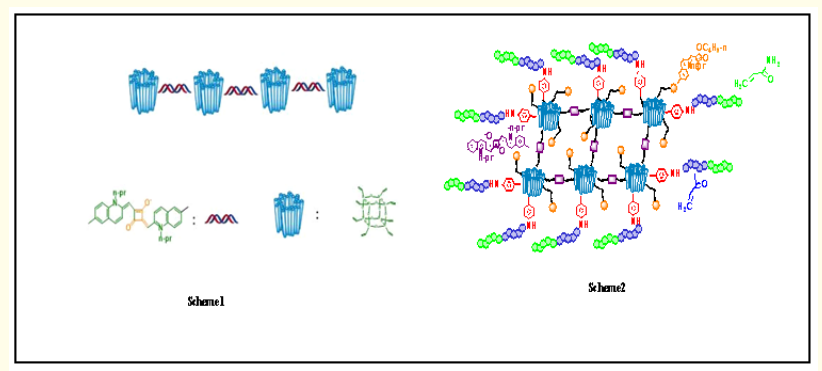

Figure a 


\section{Experimental}

\section{Synthesis of OV-POSS-Squaraine}

OV-POSS-Squaraine was prepared in $50 \mathrm{~mL}$ sealed three necked bottle the reactions were carried out under a nitrogen atmosphere, using a vacuum line system. Added a mixture of Squaraine $121 \mathrm{mg}$ [16], followed by addition OVPOSS $127 \mathrm{mg}$ and palladium acetate $16 \mathrm{mg}$ and $\mathrm{K}_{2} \mathrm{CO}_{3} 83 \mathrm{mg}$ the bottle was evacuated under vacuum and then flushed with dry nitrogen three times. Then N-methyl pyrrolidone was added, the reaction mixture was refluxed at $120^{\circ} \mathrm{C}$ under nitrogen for $12 \mathrm{~h}$ and then cooled to room temperature. The mixture was diluted with $50 \mathrm{~mL}$ of water and faltered. The precipitate was washed with toluene first, and then dissolved in DMSO. The DMSO solution was added drop wise into $100 \mathrm{~mL} \mathrm{H}_{2} \mathrm{O}$ the purification procedure was repeated three times [16].

Preparation of hybrid OV-POSS co-poly acryl amide

It was prepared by free-radical polymerization. In a $50 \mathrm{~mL}$ round bottom flask, OV-POSS amine acryl amide (0.223 g), acryl amide and AIBN (0.029 g, $0.15 \mathrm{mmol}$ ) were dissolved in DMF (16 $\mathrm{mL}$ ) under a nitrogen atmosphere and stirred at $70^{\circ} \mathrm{C}$.Finally, the solution was dropped into excess toluene to precipitate the copolymer and dissolved in ethyl acetate/petroleum ether (v/ $\mathrm{v}=1: 1$ ) [17].

\section{Results and Discussion}

\section{Aggregation effect characterization of Squaraine}

The aggregation mechanism is classified as either J-type or H-type. In this regard, the absorption spectra of SQ without OVPOSS were shown in Figure 1(a) and SQ with OV-POSS is shown in Figure 1(b). DMSO and DMSO-water solvents with a volume ratio at $1: 1$ and $1: 9$ are recorded. The SQ without OV-POSS shows a big aggregation in the mixed solvent. This result means that the optical properties of SQ without OV-POSS are not stable in a poor solvent, even in a mixed solution containing a small fraction of poor solution. But the SQ with OV-POSS there was no more change in the absorbance which indicates that there is no aggregation and stable in a poor solvent.

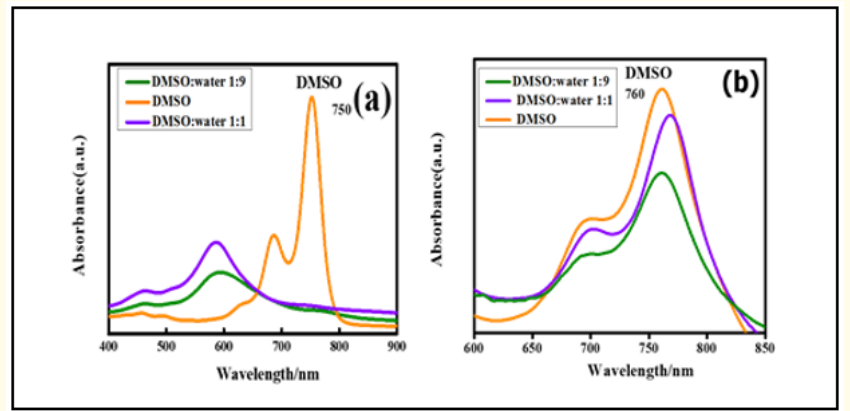

Figure 1: (a) the absorption spectra in DMSO and DMSO-water of SQ without OV-POSS and (b) SQ with OV-POSS.

\section{Aggregation effect characterization of co-poly acryl amide}

Similarly, the fluorescence was inclined to decrease significantly for the pristine co-poly acryl amide without OV-POSS. This quenching is due to the interaction between the organic luminophore and THF molecules which THF is a poor solvent and water is a good solvent Figure 2 (a). When it was incorporated with OV-POSS Figure 2 (b), the aggregation effect was weakened to a great extent due to the steric hindrance functional group of OV-POSS. As shown in the inset images, the film surfaces of co-poly acryl amide containing OV-POSS was quite smooth. However, the surface of pristine co-polyacrylamide was exhibited observable cracks and molecule aggregation indicating that the OV-POSS cage can effectively improve the ability of film formation and prevent aggregation.

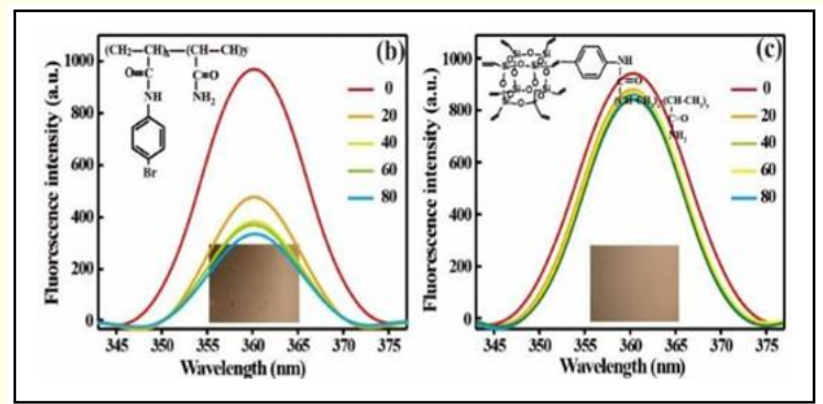

Figure 2: (a) co-poly acrylamide without OV-POSS (b) co-poly (acrylamide) with OV-POSS in THF-water solvent with different THF content. 
Morphology of OV-POSS-SQ and OV-POSS-co-poly acryl amide

Field emission scanning electron microscope FE-SEM images show in Figure 3 image of OV-POSS it looks like cubic shape, Figure 3 (a) the morphology of OV-POSS-Squaraine was formed homogenous, the resultant was obtained; in Figure 3 (b) OVPOSS co-poly acryl amide shows a smooth surface and better compatibility. It demonstrates that OV-POSS plays an important role in the interfacial strength and interfacial compatibility, which might be referred to the lubricating effects of OV-POSS particles and making the molecular movement easily, detect that the dispersed phase was reduced in the presence of OV-POSS. As a result, a good compatibility and miscibility were obtained in the OV-POSS-Squaraine and OV-POSS co-poly acrylamide.

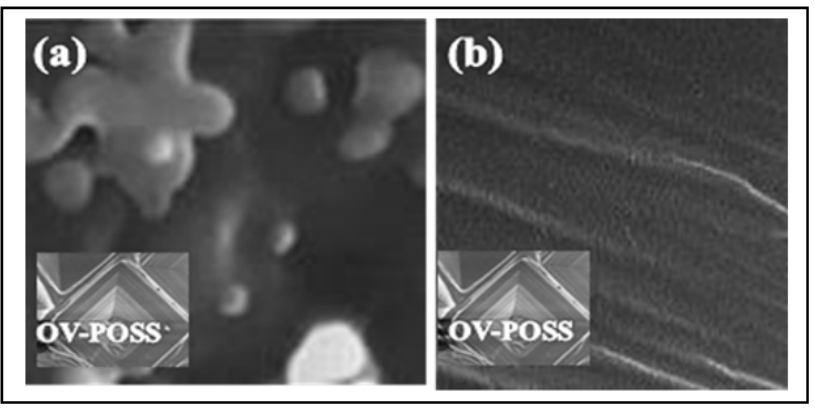

Figure 3: (a) FE-SEM images of (a) POSS-SQ(b) OV-POSS co-poly acrylamide.

\section{Conclusion}

The traditional systems containing Squaraine and poly acryl amide were suffering of aggregation effect and compatibility which traditional materials are suffering from it when applied in many fields so we have highlighted recent progress towards the development of these two important systems OV-POSS-Squaraine and OV-POSS-co-poly acryl amide designed and prepared successfully. Our work has opened new road for the novelty of new synthesis

\section{Acknowledgments}

The authors gratefully acknowledge financial support from the National Natural Science Foundation of China (Grant Nos. 21471030, 21671037, and 21771036).

\section{Bibliography}

1. Wang Z., et al. "Synthesis and properties of two novel copolymers based on squaraine and fluorene units for solar cell materials". Chinese Chemical Letters 22.8 (2011): 1001-1004.

2. Bagnis D., et al. "Marked alkyl-vs alkenyl-substitutent effects on squaraine dye solid-state structure, carrier mobility, and bulk-heterojunction solar cell efficiency". Journal of the American Chemical Society 132.10 (2010): 4074-4075.

3. Yan Z., et al. "A Convenient Organic-Inorganic Hybrid Approach Toward Highly Stable Squaraine Dyes with Reduced H-Aggregation”. Advanced Functional Materials 22.2 (2012): 345-352.

4. Yan Z., et al. "An effective real-time colorimeteric sensor for sensitive and selective detection of cysteine under physiological conditions". Analyst 136.9 (2011): 1916-1921.

5. Puyad AL., et al. "A comparative study of semi-squaraine and squaraine dyes using computational techniques: tuning the charge transfer/biradicaloid character by substitution". Journal of molecular modeling 19.1 (2013): 275-287.

6. Yan Z., et al. "Near-infrared absorbing squaraine dyes for solar cells: relationship between architecture and performance". The Journal of Physical Chemistry C 116.16 (2012): 8894-8900.

7. TM Geng and DY Wu. Luminescence 30 (2015): 1263-1268.

8. Xu H., et al. "Preparation, Thermal Properties, and T g Increase Mechanism of Poly (acetoxystyrene-co-octavinyl-polyhedral oligomeric silsesquioxane) Hybrid Nanocomposites". Macromolecules 8.25 (2005): 10455-10460.

9. Su X., et al. "Controllable preparation and optical limiting properties of POSS-based functional hybrid nanocomposites with different molecular architectures". Macromolecules 42.22 (2009): 8969-8976.

10. Yang Bh., et al. "Design and architecture of low-dielectric-constant organic-inorganic hybrids from octahydridosilsesquioxanes". Journal of Materials Chemistry 19.47 (2009): 9038-9044.

11. Su X., et al. "Molecular hybrid optical limiting materials from polyhedral oligomer silsequioxane: preparation and relationship between molecular structure and properties". Macromolecules 43.6 (2010): 2840-2845. 
12. Cordes., et al. "Recent developments in the chemistry of cubic polyhedral oligosilsesquioxanes". Chemical Review 110.4 (2010): 2081-2173.

13. Wang X., et al. "Preparation and properties of electron injecting molecular hybrid materials with high thermal performance". Journal of Materials Chemistry 21.34 (2011): 1294112948.

14. Su X., et al. "The preparation and optical limiting properties of POSS-based molecular hybrid functional materials". Dyes and Pigments 87.1 (2010): 69-75.

15. Omer N., et al. "A facile strategy for preparation and application of squaraine nanometer hybrids with broad band absorption". Materials Letters 238 (2019): 107-111.

16. Omer N., et al. "Highly selective chemo-sensor for repetitive detection of $\mathrm{Fe} 3+$ in pure water and bio-imaging". Analyst (2019).

17. Y Zhu., et al. J. Mater. Chem. C, 1 (2013): 5277-5284.

\section{Volume 3 Issue 8 August 2019}

(C) All rights are reserved by Nahla Omer and Hongyao

Xu. 